\title{
Development of Broadband LHCP Pyramidal Horn Antenna with Septum Gaussian Profile Polarizer for CP-SAR Sensor Onboard Microsatellite
}

\author{
Agus Hendra Wahyudi',2, Cahya Edi Santosa ${ }^{1,2}$, Josaphat Tetuko Sri Sumantyo ${ }^{1}$ \\ ${ }^{1} J o s a p h a t$ Microwave Remote Sensing Lab, Chiba University, Chiba, Japan \\ ${ }^{2}$ Indonesian National Institute of Aeronautics and Space (LAPAN), DKI Jakarta, Indonesia \\ Email: agus8hendra@gmail.com
}

How to cite this paper: Wahyudi, A.H., Santosa, C.E. and Sumantyo, J.T.S. (2018) Development of Broadband LHCP Pyramidal Horn Antenna with Septum Gaussian Profile Polarizer for CP-SAR Sensor Onboard Microsatellite. Open Journal of Antennas and Propagation, 6, 73-83.

https://doi.org/10.4236/ojapr.2018.64007

Received: October 22, 2018

Accepted: November 26, 2018

Published: November 29, 2018

Copyright $\odot 2018$ by authors and Scientific Research Publishing Inc. This work is licensed under the Creative Commons Attribution International License (CC BY 4.0).

http://creativecommons.org/licenses/by/4.0/

cC) (7) Open Access

\begin{abstract}
Horn Antenna has many applications such as communication, radar, and standard reference antenna for measurement. In this research, we designed a pyramidal horn for a Circularly Polarized Synthetic Aperture Radar (CP-SAR) sensor onboard a microsatellite. We utilized a 3D printer with Fused Deposition Modelling (FDM) technology for fast, low-cost, and low-weight production. Polylactide (PLA) material was used to construct 3D structures, and a copper conductive coating was painted on its surface. Gaussian distribution function was employed to create a septum polarizer profile. NPC-220 A with 1.6 thickness and 2.17 dielectric constant was used to make a microstrip monopole antenna and stripline feeding to feed the pyramidal horn to generate TE01 mode at one side of the waveguide. The design, parametric studies, and measurements are discussed in this paper. The designed antenna can achieve wide bandwidth $28 \%$ of $3 \mathrm{~dB}$ axial ratio, and more than $22 \%$ of $\mathrm{s} 11 \leq-10 \mathrm{~dB}$ in working frequency that is acceptable for CP-SAR requirement on the microsatellite.
\end{abstract}

\section{Keywords}

CP-SAR, Microsatellite, Pyramidal Horn, 3D Printer

\section{Introduction}

Horn antenna is important for many applications such as communication, radar, laboratory's standard reference antenna. Also, a simple design, wide bandwidth, narrow beam-width, and high-gain characteristic are also required for its usage 
in many applications, for example, airborne radar altimeters, and microsatellite parabolic-feeding antennas are some common applications of the horn antenna.

A circularly polarized horn antenna has some advantages than a linearly polarized one regarding losses due to ionosphere's Faraday effects and platform movement polarization mismatch [1]. Many methods have been proposed to produce the circularly polarized horn antenna. Some researchers designed stepped septum inside square waveguide as in [2] [3] [4] to achieve wide bandwidth axial ratio, while a different function was used to design septum polarizer to modify stepped septum transition with smooth lines such as Legendre function and Sigmoid function [5] [6]. However, they still have many parameters, and the lines are not easy to fabricate using the FDM 3D printer. Another method used microstrip substrate dielectric metamaterials in front of the aperture to make orthogonal waves with equal amplitude and 90-degree phase shift [7] [8] which required more structure volume and weight to provide. Also, in [9] [10] a crossed-dipole metasurface slot is used to change the linearly polarized to circularly polarized wave on the waveguide for horn antenna. There are still many parameters and add a little weight to the metal horn standard antenna structure, and the axial ratio bandwidth also does not meet the needs of our CP-SAR design system (less than $8 \%$ ).

This research proposed a Gaussian distribution function as the profile of septum polarizer. The polarizer inside the square waveguide is able to generate wideband circularly polarized waves in front of a pyramidal horn antenna particularly in X band for a CP-SAR microsatellite sensor. The design used fewer parameters and was easy to fabricate. A $3 \mathrm{D}$ printing technique was used to fabricate the structure to achieve low-weight, low-cost, and fast prototyping. The surface of the antenna was coated with a copper conductive coating. Stripline feeding with a strip monopole was designed to feed one side of the waveguide to generate TE01 mode of linearly polarized waves. The design, simulation, parametric study, and measurement are described in this paper.

\section{Antenna Design and Simulation}

\subsection{Horn Pyramidal Design}

The pyramidal horn antenna can be designed at a required gain and a certain frequency. Firstly, cutoff frequency was chosen at $6.8 \mathrm{GHz}$ to determine the waveguide dimension. Then the optimum gain method was used to calculate the pyramidal horn dimension in axial length, horn's aperture width, and height as in [11] [12].

The antenna design was simulated in a CST microwave studio software. Next, fabrication of horn, waveguide, the Gaussian polarizer, and the cavity were used with $1.75 \mathrm{~mm}$ polylactide (PLA) materials, with $0.2 \mathrm{~mm}$ slice resolution. A Scoovo C170 3D printer with $0.3 \mathrm{~mm}$ extruder was utilized to print all 3D structures at $220 \mathrm{O}$ Celsius. The design's flowchart is illustrated in Figure 1 below.

As shown in Figure 1, after the 3D-printed structure was done, finishing treatments were needed to make a smoother surface with sandpaper no. 1000, 


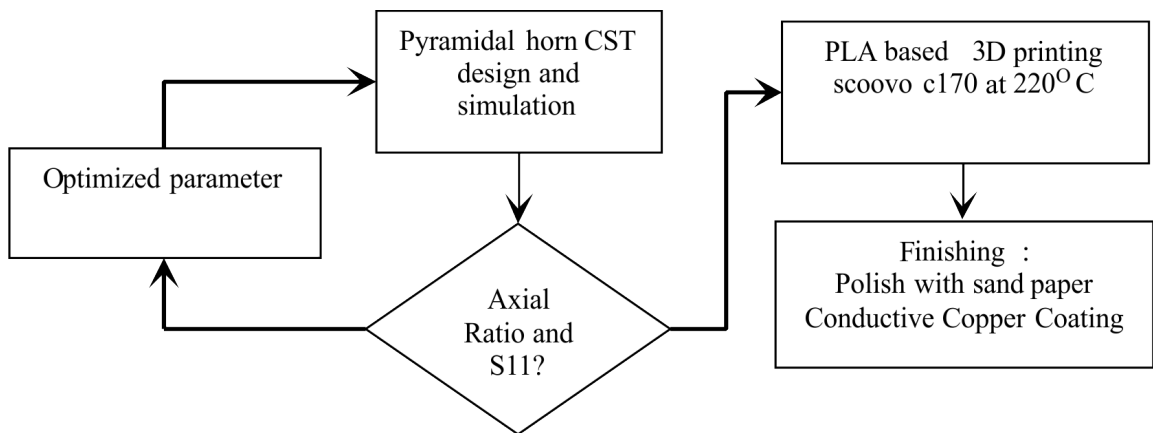

Figure 1. Design's flowchart.

and it was later metalized with conductive copper paint with three-layer thickness. The designed and finished, a fabricated pyramidal horn antenna is shown in Figure 2.

PLA structure was characterized by measuring its dielectric constant with 85070e dielectric probe kit. Short, air and water calibrations were done before measurement. The result is shown in Figure 3, in which the PLA structures has 2.095 dielectric constant and 0.0457 loss tangent.

\subsection{Septum Gaussian Profile Polarizer}

A Septum polarizer inside the square waveguide was designed to modify the linearly polarized wave to become circularly polarized one. The polarizer profile can be shaped with normal distribution or Gaussian distribution, where probability mass density function has the form [10] [13]:

$$
P(x)=\frac{1}{\sqrt{2 \pi \sigma}} \mathrm{e}^{-(x-\mu)^{2} / 2 \sigma^{2}}
$$

We added a variable to adjust its Gaussian septum amplitude:

$$
P(x)=A \frac{1}{\sqrt{2 \pi \sigma}} \mathrm{e}^{-(x-\mu)^{2} / 2 \sigma^{2}}
$$

where $x$ is a random variable, $A$ is the Gaussian amplitude, $\mu$ is mean, and $\sigma$ is the standard deviation. By controlling these parameters, the polarizer shows wide bandwidth in an axial ratio $\leq 3 \mathrm{~dB}$ where an orthogonal wave with equal amplitude and 90-degree phase shift was evidenced.

\subsection{Design of Stripline Feeding}

Stripline feeding inside the waveguide was designed as the monopole antenna which generates TE01 mode at one side of the waveguide. The substrate of NPC-220A with a dielectric constant of 2.17 and $1.6 \mathrm{~mm}$ thickness was used. An ellipse monopole shape was chosen as shown in Figure 4. Initially, the length (ellipse E-plane diameter) and width of stripline were obtained by the following equation:

$$
L=\frac{\lambda_{g}}{4}
$$




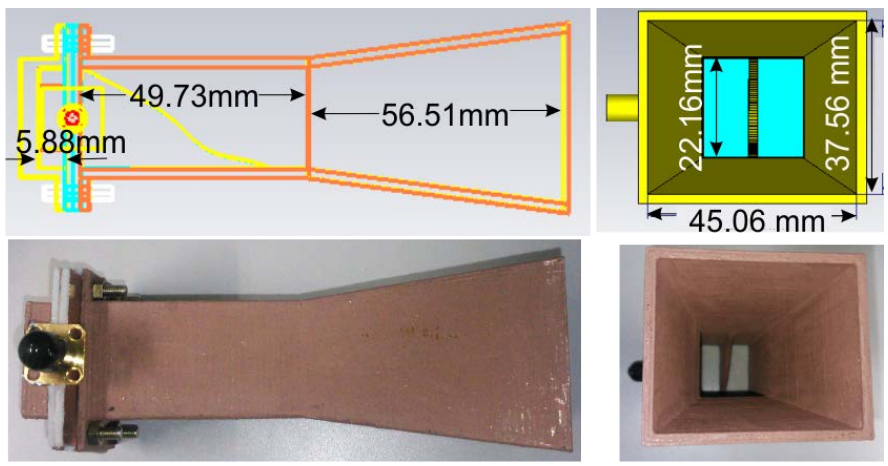

Figure 2. The designed and finished 3D printed LHCP pyramidal Horn antenna.

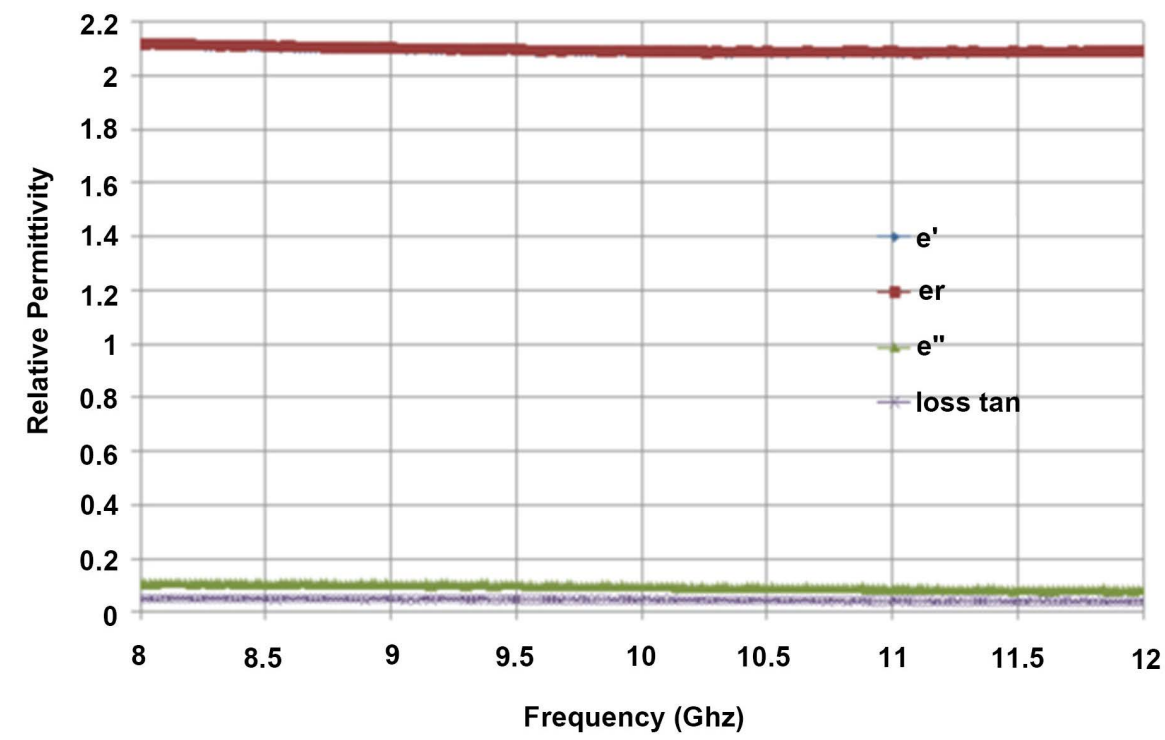

Figure 3. PLA structures Horn antenna dielectric constant measurement.

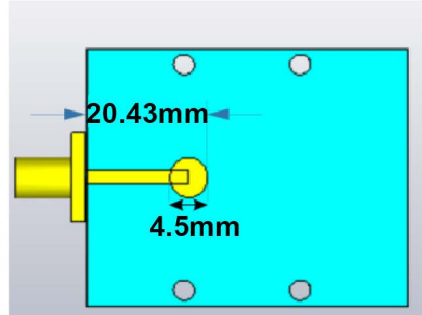

(a) middle feeding

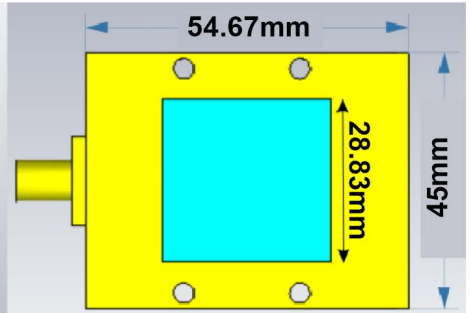

(b) botton and top ground

Figure 4. Feeding stripline and ellipse Monopole.

$$
\begin{gathered}
\lambda_{g}=\frac{\lambda_{0}}{\sqrt{\varepsilon r}} \\
Z_{0}=\frac{60}{\sqrt{\varepsilon r}} \ln \frac{1.9(2 h+t)}{0.8 \omega+t}
\end{gathered}
$$

where $h$ is substrate thickness of $1.6 \mathrm{~mm}, \mathrm{t}$ is the copper thickness of $0.035 \mathrm{~mm}$, $\mathrm{w}$ is the width of the stripline, and $\mathrm{r}$ the dielectric constant substrate NPC-220 
2.17. In this research, we use an ellipse monopole strip shape to have broadband bandwidth [14] which was almost similar to a balloon shape which had the intermediate bandwidth in [11].

\section{Parametric Studies}

\subsection{Effect of Gaussian Mean Value}

The effect of the Gaussian mean value was simulated at three points i.e. 23.2695, 33.2695 and $43.2695 \mathrm{~mm}$. It is depicted in the image of the simulation's results in Figure 5 that the bandwidth's axial ratio less than $3 \mathrm{~dB}$, reaching its optimal at the peak position at $33.2695 \mathrm{~mm}$, starting from $8.05 \mathrm{GHz}$ or more than $39 \%$.

\subsection{Effect of Gaussian Standard Deviation}

The variation of standard deviation for Gaussian distribution value at 5.2, 6.2, 7.2 and 8.2 was simulated. The effect of standard deviation to axial ratio is illustrated in Figure 6. Standard deviation 6.2 was chosen because it had a wideband axial ratio below $2 \mathrm{~dB}$ from $8.5 \mathrm{GHz}$ or more than $34 \%$.

\subsection{Effect of Gaussian Amplitude Scale}

A simulation of Gaussian scale amplitude on three values 28.48, 48.48, and 68.48 was performed. The results are shown in Figure 7. The larger the scale would give greater Gaussian peak value, and the width of the axial ratio below $3 \mathrm{~dB}$ decreased. The best value was achieved in a equals 48.48 where the axial ratio was less than $3 \mathrm{~dB}$, starting at 8.05 or more than $38 \%$.

\subsection{Effect of Feeding Length to S11}

The simulation of feeding lengths has been varied from 3.2, 4.5 and $5.2 \mathrm{~mm}$. The effect of S11 is shown in Figure 8, in which with $4.5 \mathrm{~mm}$ feeding length and 2 $\mathrm{mm}$ feeding width, the best resonance happened at $9.2 \mathrm{GHz}$ or more than $29 \%$.

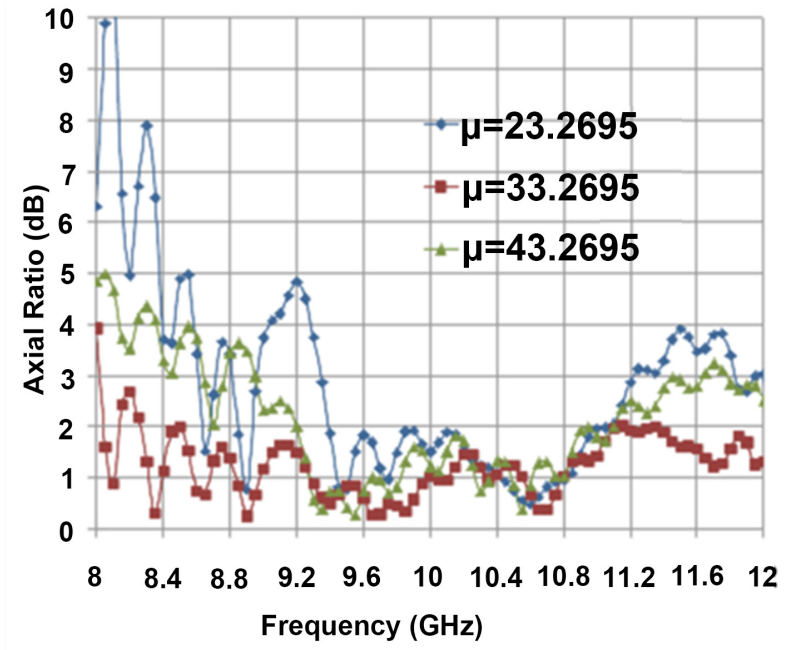

Figure 5. Effect of Gaussian mean value to axial ratio. 


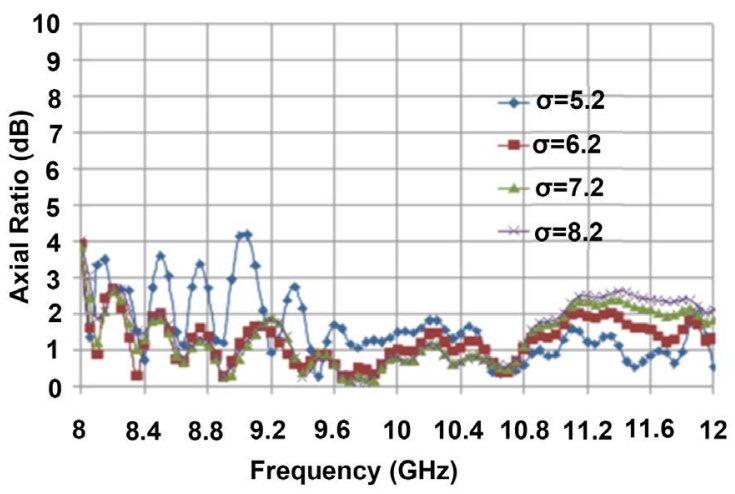

Figure 6. Effect of standard deviation value to axial ratio.

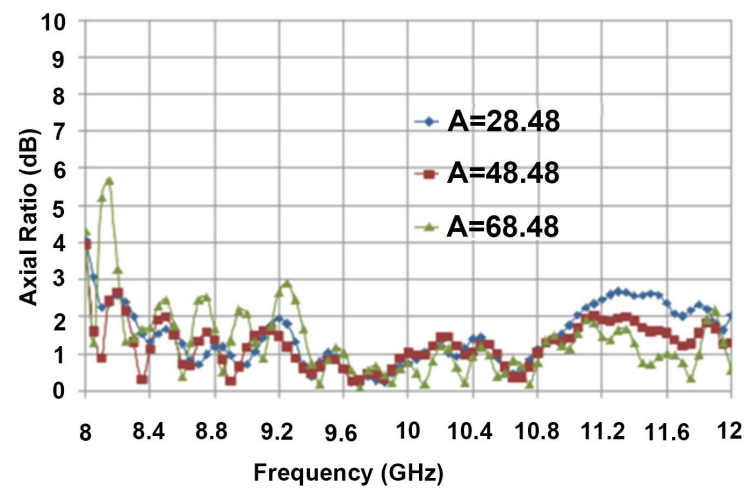

Figure 7. Effect of Gaussian amplitude scale value to axial ratio.

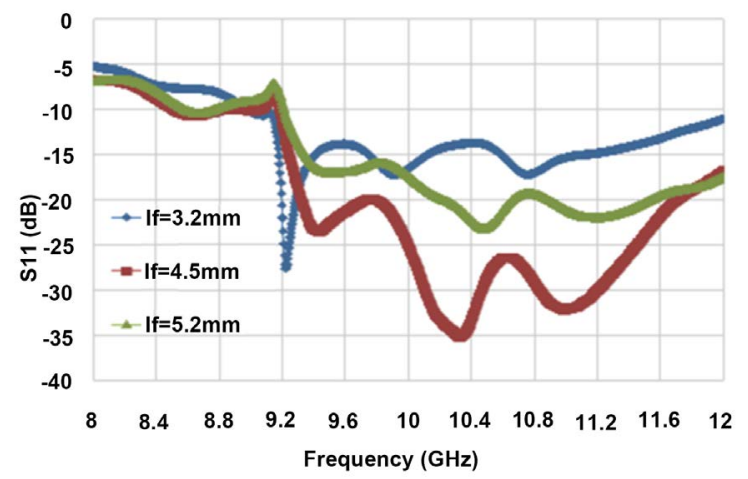

Figure 8. Effect of feeding length to S11.

From above parametric studies and horn optimum gain method, we chose final design Gaussian circularly polarized pyramidal horn as the following Table 1 to get very wide axial ratio and impedance bandwidth.

\section{Measurement Result and Discussion}

We measured the fabricated pyramidal horn antenna inside an anechoic chamber with VNA Agilent and turn-table controller as shown in Figure 9. The comparisons between simulation and measurement are presented in Figures 10-13 for S11, axial ratio, gain and radiation pattern at $9.6 \mathrm{GHz}$ respectively. 
Table 1. Optimized pyramidal horn with Gaussian profile polarizer design's parameters.

\begin{tabular}{cccccc}
\hline$\mu$ & $\sigma$ & A & Lf & Horn's Aperture & Horn's Axial Length \\
\hline 33.269 & 6.2 & 48.48 & $4.5 \mathrm{~mm}$ & $45 \times 37.5 \mathrm{~mm}$ & $50 \mathrm{~mm}$ \\
\hline
\end{tabular}

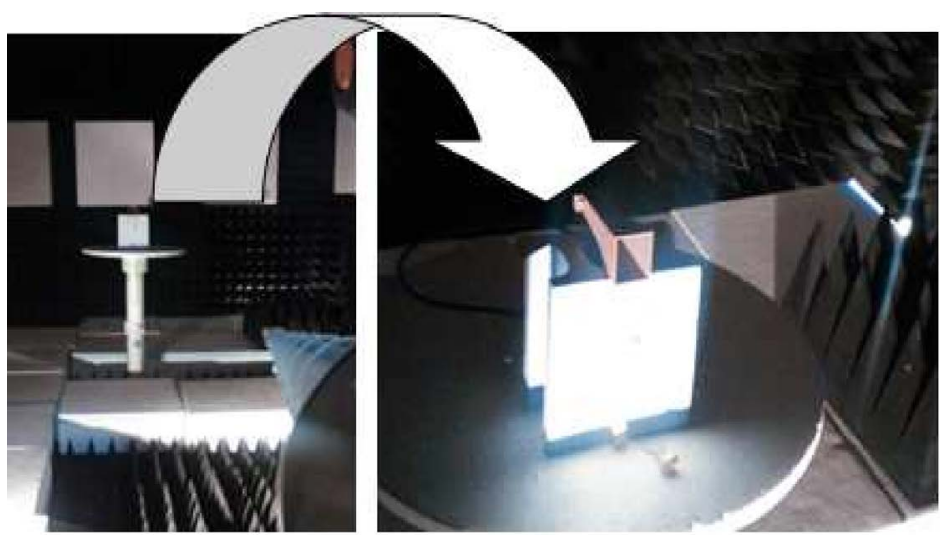

Figure 9. Measurement of the pyramidal Horn antenna in anechoic chamber.

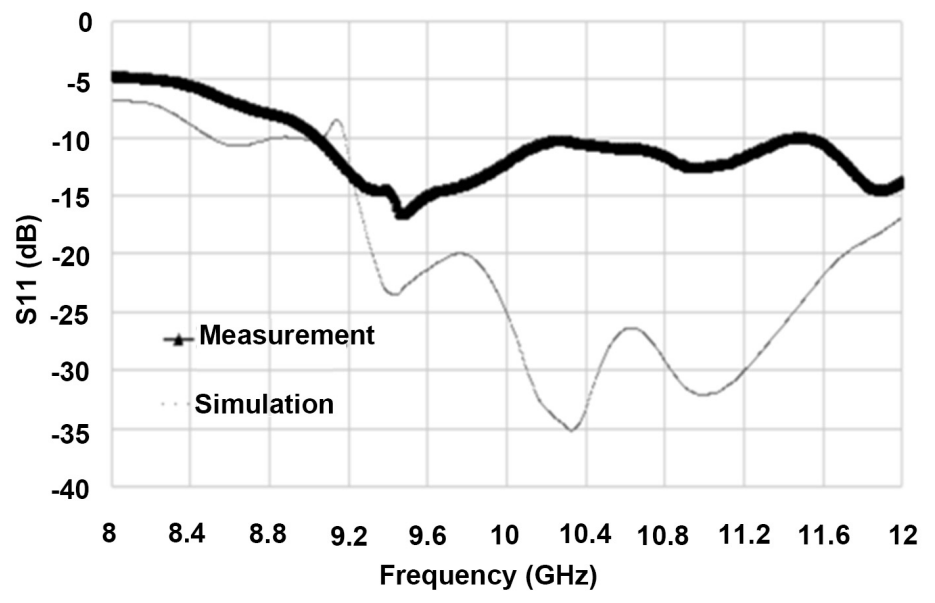

Figure 10. Reflection coefficient (S11) vs frequency.

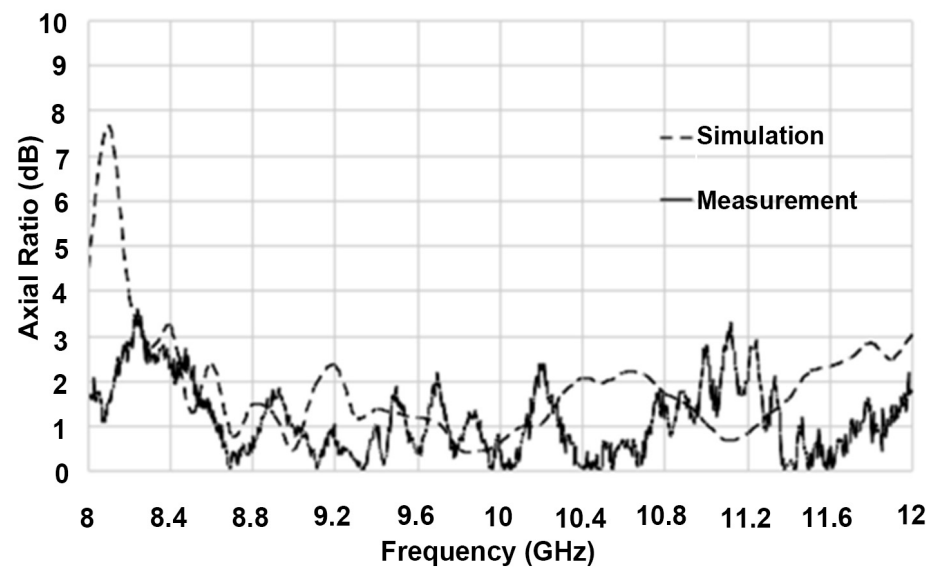

Figure 11. Axial ratio vs frequency. 


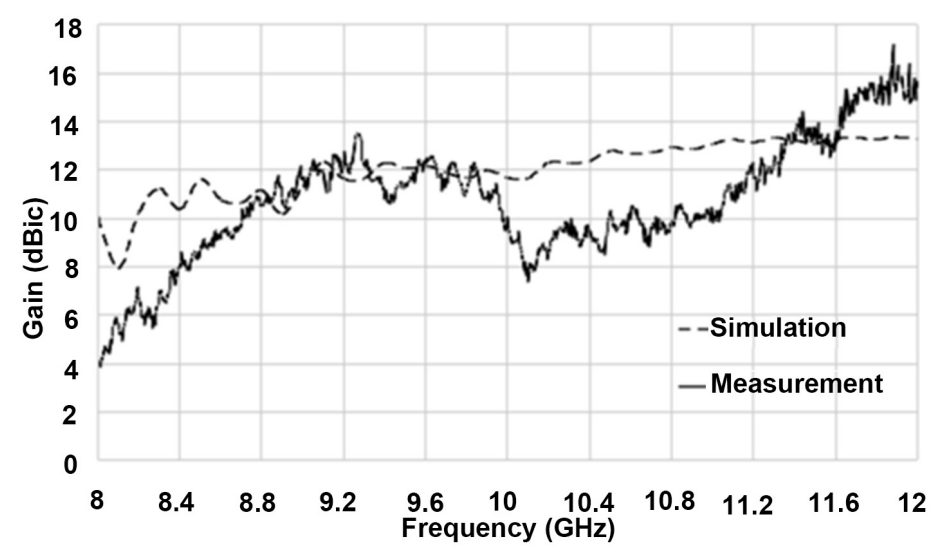

Figure 12. Gain vs frequency.

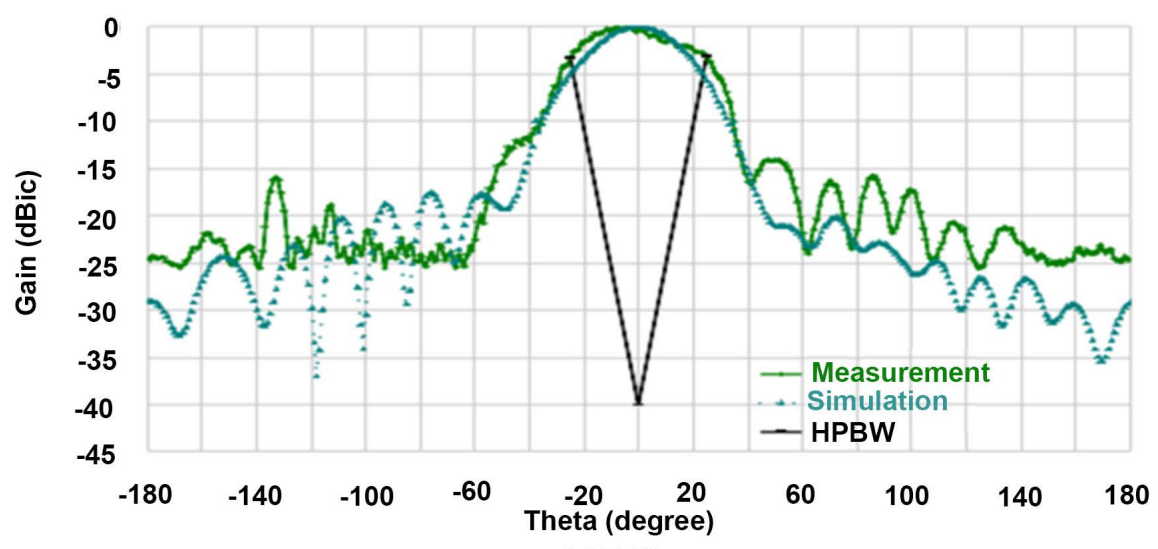

(a) E-Plane

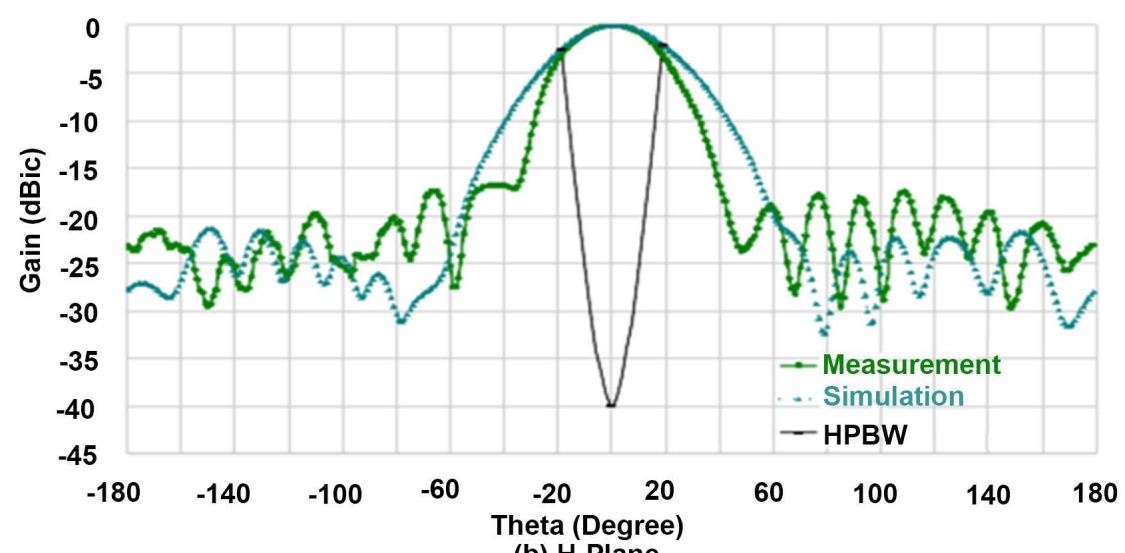

(b) H-Plane

Figure 13. Gain radiation pattern at $9.6 \mathrm{GHz}$.

Reflection coefficient S11 in Figure 10 shows that the antenna has a wide bandwidth from 9.2 to $11.4 \mathrm{GHz}$ in measurement, or fractional bandwidth S11 is below $-10 \mathrm{~dB}$ more than $22 \%$. This value is comparable with simulation even though better matching impedance $\mathrm{S} 11$ below $-10 \mathrm{~dB}$ wider in the simulation can be achieved. The difference in reflection coefficients between measurements and simulations is caused by imperfect fabrication and assembly, for example, the distance between the upper and lower substrate is not fixed, and straight, 
slightly more or less etching on the stripline and ground, and losses due to the surface resistance of 3D printed horn. However, it also fulfills our $\mathrm{X}$ band CP-SAR onboard microsatellite's bandwidth requirement which is only 800 $\mathrm{MHz}(8 \%)$.

The axial ratio in Figure 11 shows the pyramidal horn antenna has a circularly polarized axial ratioless than $3 \mathrm{~dB}$ from 8.3 until $11.1 \mathrm{GHz}$ or more than $25 \%$ fractional bandwidth, which is comparable with simulation's result.

Figure 12 shows gain vs. frequency, where we can see that gain antenna can achieve $8-14 \mathrm{dBic}$ in working frequency. $12 \mathrm{dBic}$ can be achieved at $9.6 \mathrm{GHz}$ particularly in the agreement between simulation and measurement. The small difference happened because of the imperfectness of fabrication and alignment of the stripline feeding, cavity, pyramidal horn, and waveguide.

Radiation pattern at $9.6 \mathrm{GHz}$ was measured in an anechoic chamber with a 1-degree resolution from the turntable controller. The result shown in Figure 13 , we can see that the simulation and measurement gain radiation pattern is in agreement. Also, half-power beam widths are 50 degrees for E-plane and 38 degrees for $\mathrm{H}$-plane, with this pyramidal horn $\mathrm{CP}$ antenna serving as important SAR parameter calculation.

When compared with previous studies [3] the gain radiation pattern of antenna measurement results are very close to the simulation, and the back lobe is very damped. The small rear lobe value in the proposed antenna is because there is no air gap between the cavity, feeding stripline and waveguide. Besides, the higher gain is due to longer horn's axial length and more upper conductive copper coating in this research than the nonpyramidal horn's axial length and conductive carbon coating material in [3].

\section{Comparison with the Previous Method}

This research has some benefits compared to previous methods as summarized in Table 2. It reduced the polarizer's parameter number and enabled easy fabrication by using the 3D printed FDM technology. Also, very wide bandwidth can be provided with this Gaussian polarizer shape compare to other methods.

\section{Conclusion}

This research proposed the pyramidal horn for Circularly Polarized Synthetic Aperture Radar (CP-SAR) onboard a microsatellite. PLA material was used to construct 3D structures, and the copper conductive coating was painted on its surface by employing a Gaussian distribution function to create the septum polarizer profile. The NPC-220 A with 1.6 thickness and 2.17 dielectric constant was used to make the microstrip monopole antenna and stripline feeding to feed the pyramidal horn to generate TE01 mode at one side of the waveguide. The proposed antenna obtained a wide bandwidth of axial ratio $\leq 3 \mathrm{~dB}$ of $28 \%$ and S11 $\leq-10 \mathrm{~dB}$ more than $22 \%$, which are acceptable for CP-SAR onboard microsatellite. In the future, array configuration with this horn designed 
Table 2. Comparison with previous methods.

\begin{tabular}{cccc}
\hline Horn Type & Polarizer & AR Bandwidth & $\begin{array}{c}\text { Parameter's } \\
\text { Number }\end{array}$ \\
\hline Pyramidal [proposed] & Gaussian Function & $28 \%$ & 4 \\
Conical [2] & Stepped septum & $18.6 \%$ & 10 \\
Pyramidal [3] & Stepped septum & $5 \%$ & 10 \\
Corrugated conical [4] & Stepped septum & $5 \%$ & 10 \\
Conical [5] [6] & Legendre, Sigmoid & $20 \% \& 25 \%$ & 9,10 \\
Pyramidal [7] & Curve chiral metamaterial & $0.8 \%$ \& 1.4\% & 7 \\
$\begin{array}{c}\text { Corrugated conical, } \\
\text { pyramidal [9] [10] }\end{array}$ & Crossed-dipoles & $2.5 \%$ \& 7.4\% & 6,10 \\
\hline
\end{tabular}

should be done to enhance gain and make pencil beam width so that this design can be used as microsatellite sensor for environmental monitoring. Another method also can be used such as using this design pyramidal horn for the feeder in focus of large reflector of CP-SAR microsatellite.

\section{Acknowledgements}

The authors thank Riset-Pro Ristek-Dikti, the Japanese Government National Budget-Ministry of Education and Technology (MEXT) FY2015-2017 under Grant 2101; Chiba University Strategic Priority Research Promotion Program FY2016-FY2018; Taiwan National Space Organization (NSPO) FY2017-2018; and Indonesian National Institute of Aeronautics and Space (LAPAN).

\section{Conflicts of Interest}

The authors declare no conflicts of interest regarding the publication of this paper.

\section{References}

[1] Gao, S., et al. (2014) Circularly Polarized Antenna. John Wiley and Son, New York. https://doi.org/10.1002/9781118790526

[2] Huang, Y., et al. (2015) A Novel CP Horn Antenna with Switchable Polarization by Single Port Feeding. International Journal of Antennas and Propagation, 2015, Article ID: 562521. https://doi.org/10.1155/2015/562521

[3] Wahyudi, A.H., et al. (2018) PLA-Based 3D Printed Circularly Polarized X-Band Horn Array Antenna for CP-SAR Sensor. IEEE 2018 International Workshop on Antenna Technology (IWAT), Nanjing, 5-7 March 2018, 1-4. https://doi.org/10.1109/IWAT.2018.8379219

[4] Chio. T.-H., et al. (2016) A 3D-Printed Compact Dual-Circularly Polarized Corrugated Horn with Integrated Septum Polarizer. Proceedings of ISAP 2016, Okinawa, 18-21 July 2016, 272-273.

[5] Kim, I., et al. (2014) Enhancing the Power Capabilities of the Stepped Septum Using an Optimized Smooth Sigmoid Profile. IEEE Antenna and Propagation Magazine, 56, 16-42. https://doi.org/10.1109/MAP.2014.6971913 
[6] Angevain, J.-C. and Fonseca, N.J.G. (2017) Waveguide Septum Polarizer Shaped with Legendre Polynomials. 11 th European Conference on Antennas and Propagation (EUCAP), Paris, 19-24 March 2017, 2286.

[7] Ma, X., et al. (2014) A Dual Circularly Polarized Horn Antenna in Ku-Band Based on Chiral Metamaterial. IEEE Transactions on Antennas and Propagation, 62, 2307-2311. https://doi.org/10.1109/TAP.2014.2301841

[8] Akgol, O., et al. (2018) Design of Metasurface Polarization Converter From Linearly Polarized Signal to Circularly Polarized Signal. Optik, 161, 12-19. https://doi.org/10.1016/j.ijleo.2018.02.028

[9] Barbuto, M., et al. (2013) A Combined Bandpass Filter and Polarization Transformer for Horn Antennas. IEEE Antennas and Wireless Propagation Letters, 12, 1065-1068. https://doi.org/10.1109/LAWP.2013.2280151

[10] Lin, C., et al. (2018) Circularly Polarized Horns Based on Standard Horns and a Metasurface Polarizer. IEEE Antennas and Wireless Propagation Letters, 17, 480-484. https://doi.org/10.1109/LAWP.2018.2796641

[11] Ballanis, C.A. (2016) Antenna Theory, Analysis and Design. John Wiley and Son, New York.

[12] Milligan, T. (1985) Modern Antenna Design. McGraw-Hill, New York.

[13] Nadarajah, S. (2005) A Generalized Normal Distribution. Taylor \& Francis, Milton Park.

[14] Kumar, G. (2013) Broadband Microstrip Antenna. Artech House, London. 